\title{
Subperiosteal Implants in Treatment of Total and Partial Edentulism - A Long Term Follow Up
}

\author{
Stefan Peev ${ }^{1}$, Elitsa Sabeva ${ }^{1}$ \\ ${ }^{1,2}$ Department of Periodontology and Dental Implantology, Medical University of Varna, Bulgaria
}

\begin{abstract}
In 73 cases with advanced alveolar bone loss we placed 93 subperiosteal implants.The implants were observed for 5 to 17 years after placement. The mean period of observation was 7,63 years. The mean survival rate of the implants for the entire observation period is $74.7 \%$. The survival rate of the subperiosteal implants for a 5-year observation period is $94.5 \%$ and the complication rate is $22.2 \%$.The survival rate of the subperiosteal implants for the entire period of observation (5-17 years) is $96 \%$.
\end{abstract}

Keywords: Subperiosteal implants

\section{Introduction}

One of the most controversial issues in the modern implantology is undoubtedly the use of subperiosteal implants for treatment of the total and partial edentulism. Intraosseal implants demonstrate extremely high success and survival rate along with good soft tissue and marginal bone stability. This makes them a preferred method for treatment of the tooth loss. On the other hand the treatment with intraosseal implants requires a sufficient bone volume. That's why a number of alveolar bone augmentations are performed, which aim is to prepare the existing bone for implant placement.

\section{Literature Survey}

Because of their invasiveness and complexity, are considered some alternatives of bone augmentation, such as inferior alveolar nerve lateralization or transposition (1), the implants with reduced diameter, implants with reduced length(2) and the subperiosteal implants. The first patented in 1938 subperiosteal implants were presented in the publication of Gustav Dahl in 1943 (3) and were promoted more widely by publication of Goldberg and Gershkoff in 1949 (4). Golec(5) observed 100 subperiosteal implants and reported $100 \%$ survival rate at the end of the fourth year, $96 \%$ at the end of the fifth and 92\% survival rate at the end of the sixth year. Mercier et al. (6) reported success rate of $60 \%$ and failure - $17 \%$. According to Young et al. (7) the survival rate for a period of 5 years is $90 \%$ and at the end of the sixth year it is $75 \%$. Bailey et al. (8) reported $86 \%$ survival rate of the subperiosteal implants for a period of 14 years. James et al. (9) in a study on 147 subperiosteal implants reported $98 \%$ survival rate at the end of the fifth year, $87 \%$ for a period of 10 years and $78 \%$ survival rate at the end of the thirteenth year. The main disadvantage of the subperiosteal implants is the principle of their retention. Although there are such techniques, the subperiosteal implants are not osseointegratable. They can not prevent the atrophy of the bone in the area, in which they are placed. Thereupon, after a period of time (5 - 30years) is observed a dehiscence of the metal framework of the implant. The most common complication is the inflammation, which is not difficult to treat, but leads to a progressive bone resorption around the implant. Often recurrent inflammations around the subperiosteal implants are an indication of its removal (10).

\section{Methods}

In 73 cases with advanced alveolar bone atrophy were performed 93 subperiosteal implants. 72 of them were placed according to the two-stage method and 21 of them-to the one stage method. In the cases of two-stage method in the first visit after the elevation of muco-periosteal flap, an impression was taken with sterile additive silicone material, of the bone in the area, where the subperiosteal implant was planned. The flap is then repositioned and sutured. The subperiosteal implants are made of cobalt-chrom-molybdenum alloy, without nickel and beryllium. Each implant is constructed of metal framework and one or more posts connected to the metal framework through transgingival part. After two weeks, the flap was elevated again, the implant was placed in the appropriate area and the flap was repositioned and sutured. The total subperiosteal implants were placed in cases of total edentulism and the partial implants in the cases of open-arch defects. The impression for fabricating a bridgework was taken two weeks after the implant placement. The subperiosteal implants was loaded three weeks after their placement. According to the one-stage method a three-dimensional image of the relevant area of the jaw was generated, using CBCT. The three-dimensional image of the bone is used to create its three-dimensional model using 3D printer-. The model is duplicated and the subperiosteal implants are model-casted. The implant is polished, and a vent for at least one fixation micro-screw was planned. The implant was sterilized and then was fixed to the bone, after elevation of muco-periosteal flap, which after the fixation is repositioned and sutured.

In patients treated with subperiosteal implants were observed for:

1) Presence of intraoperative complications

2) Presence or absence of inflammation in the implant area

3) Exposure of the framework of the implant

4) Presence or absence of bone resorption around the implant, which is visible on radiography

5) The survival rate of the implants and the frequency of complications for a period of at least 5 years. 


\section{International Journal of Science and Research (IJSR) \\ ISSN (Online): 2319-7064 \\ Index Copernicus Value (2013): 6.14 | Impact Factor (2014): 5.611}

\section{Results}

$87,4 \%$ of the subperiosteal implants were partial and $10,5 \%-$ total. $50.5 \%$ of the implants were made with smooth surface, treated consecutively with mechanical polishing and electropolishing, and $47.4 \%$ - with a rough surface.The mean survival rate of the implants for the entire observation period is $74.7 \%$. $18.9 \%$ of the implants are failed and the partially removed implants are $4.2 \%$. The survival rate of the implants with smooth surface for the entire period is $96 \%$ and of the implants with rough surface $-64 \%$. The survival rate of two-stage implants is $76 \%$, the survival rate of one-stage implants is $95 \%$. The complication rate for the entire period is $48.4 \%$. In $29.5 \%$ of the cases bone resorption and / or exposure of the metal framework of the implant is observed, and in $16,8 \%$ of cases inflammation with or without bone loss was observed. The complication rate of implants with a smooth surface is $18.6 \%$, while at rough-surface implants it is $75 \%$. The survival rate of the subperiosteal implants for a 5 -year observation period is $94.5 \%$ and the complication rate is $22.2 \%$.

\section{Discussion}

The survival rate of the subperiosteal implants for five years is $94,5 \%$. Our result is similar to this written by Bodine $(11,12)$ and Golec(5). The survival rate for the period of observation is $74,4 \%$. The survival rate of the implants with smooth surface is $96 \%$ for the entire period of observation (5-17years). Significant difference was observed between the success rate, respectively the complication rate and the survival rate of the subperiosteal implants with smooth and with rough surface. This confirms the results reported by Surovas (13). The survival rate of two-stage implants is 76\% and the survival rate of one-stage implants is $95 \%$. It should be noted, that the one-stage method was performed only with subperiosteal implants with smooth surface, which probably influenced the survival rate in these cases. The major disadvantage of the subperiosteal implants is the more sufisticated clinical and lab procedure, which requires special training of the clinical and laboratory staff.

\section{Conclusion}

The survival rate of the subperiosteal implants for the entire period of observation (5-17 years) is 96\%. This outcome confirms that the subperiosteal implants could be reliable alternative of the two-stage method intraosseal implants in combination with major bone augmentation of the alveolar ridge.

\section{References}

[1] Peev S,Ivanov B, Sabeva E, Georgiev T, Five-Year Follow-Up of Implants Placed Simultaneously with Inferior Alveolar Nerve Lateralisation or Transposition,Scripta Scientifica Medicinae Dentalis, vol. 1, No2, 2015, pp. 44-48

[2] Peev S, Sabeva E, Gusiyska A, Georgiev T, Tonchev T, Long-term follow-up of Implants with Reduced Length.Scripta Scientifica Medicinae Dentalis, vol. 1, No2, 2015, pp. 49-52
[3] Dahl, G. Om mojligheten for inplantation i ka“ken av metallskelett som bas eller retention fo" $\mathrm{r}$ fasta eller avtagbaraproteser. Odontologisk Tidskrift 1943, 51: 440449.

[4] Goldberg, N.I. \& Gershkoff, A. The implant lower denture. Dental Digest 1949, 55: 490-495.

[5] Golec, T.S. The mandibular full subperiosteal implant clinical review of 100 cases. Dental Survey 1980, 56: 32-38.

[6] Mercier, P., Cholewa, J. \& Djokovic, S. Mandibular subperiosteal implants. Journal of the Canadian Dental Association 1981, 47: 46-51.

[7] Young, L., Michel, J.D. \& Moore, D.J. A twenty-year evaluation of subperiosteal implants. Journal of Prosthetic Dentistry 1983, 49: 690-694.

[8] Bailey, J.H., Yanase, R.T. \& Bodine, R.L. The mandibu-lar subperiosteal implant denture: A fourteen-year study.Journal of Prosthetic Dentistry 1988, 60: 358361.

[9] James, R.A., Lozada, J.L., Truitt, P.H., Foust, B.E. \& Jovanovic, S.A. Subperiosteal implants. Journal of the California Dental Association 1988, 16: 10-14.

[10] Schou S, Pallesen L, Hjørting-Hansen E, Pedersen CS, Fibæk B. A 41-year history of a mandibular subperiosteal implant.Clin Oral Impl Res 2000: 11: 171-178.

[11] Bodine, R.L. Implant dentures: Follow-up after seven to ten years. Journal of the American Dental Association1963, 67: 352-363.

[12] Bodine, R.L. Evaluation of 27 mandibular subperiosteal implant dentures after 15 to 22 years. Journal of Prosthetic Dentistry 1974, 32: 188-197.

[13] Surovas O., Basics of the construction of subperiosteal implants (SPI). Novosti v stomatologii ,2011

\section{Author Profile}

Stefan Peev DMD, PhD, DSc - Head of Department of Periodontology and Dental Implantology, Medical University of Varna, Bulgaria, Director of University Dental Clinic - Varna

Elitsa Sabeva DMD - Assistant Professor at Department of Periodontology and Dental Implantology, Medical University of Varna, Bulgaria 